Stud. Univ. Babeş-Bolyai Math. 64(2019), No. 4, 565-580

DOI: $10.24193 /$ subbmath.2019.4.10

\title{
Ascent, descent and additive preserving problems
}

\author{
Mourad Oudghiri and Khalid Souilah
}

\begin{abstract}
Given an integer $n \geq 1$, we provide a complete description of all additive surjective maps, on the algebra of all bounded linear operators acting on a complex separable infinite-dimensional Hilbert space, preserving in both directions the set of all bounded linear operators with ascent (resp. descent) nongreater than $n$. In the context of Banach spaces, we consider the additive preserving problem for semi-Fredholm operators with ascent or descent non-greater than $n$.
\end{abstract}

Mathematics Subject Classification (2010): 47B49, 47L99, 47A55, 47B37.

Keywords: Linear preserver problems, ascent, descent, semi-Fredholm operators.

\section{Introduction}

Let $X$ be an infinite-dimensional Banach space over the real or complex field $\mathbb{K}$, and let $\mathcal{B}(X)$ be the algebra of all bounded linear operators on $X$.

For a subset $\Lambda \subset \mathcal{B}(X)$, we say that a map $\Phi$ on $\mathcal{B}(X)$ preserves $\Lambda$ in both directions (or, equivalently, that $\Phi$ is a preserver of $\Lambda$ in both directions) if for every $T \in \mathcal{B}(X)$,

$$
T \in \Lambda \text { if and only if } \Phi(T) \in \Lambda \text {. }
$$

For an operator $T \in \mathcal{B}(X)$, write $\operatorname{ker}(T)$ for its kernel, $\operatorname{ran}(T)$ for its range and $T^{*}$ for its adjoint on the topological dual space $X^{*}$. The ascent $\mathrm{a}(T)$ and descent $\mathrm{d}(T)$ of $T \in \mathcal{B}(X)$ are defined by

$$
\mathrm{a}(T)=\inf \left\{k \geq 0: \operatorname{ker}\left(T^{k}\right)=\operatorname{ker}\left(T^{k+1}\right)\right\}
$$

and

$$
\mathrm{d}(T)=\inf \left\{k \geq 0: \operatorname{ran}\left(T^{k}\right)=\operatorname{ran}\left(T^{k+1}\right)\right\},
$$

where the infimum over the empty set is taken to be infinite (see [15, 19]). Clearly, a bounded linear operator is injective (resp. surjective) if and only if its ascent (resp. descent) is zero.

Over the last years, there has been a considerable interest in the so-called linear preserver problems that concern the question of determining the form of all linear, or 
additive, maps on $\mathcal{B}(X)$ that leave invariant certain subsets. The most linear preserver problems were solved in the finite-dimensional context, and extended later to the infinite-dimensional one. For excellent expositions on linear preserver problems, the reader is referred to $[7,11,12,13,16]$ and the references therein.

One of the most famous problems in this direction is Kaplansky's problem [8], asking whether bijective unital linear maps $\Phi$, between semi-simple Banach algebras, preserving in both directions invertibility, are Jordan isomorphisms (i.e. $\Phi\left(a^{2}\right)=$ $\Phi(a)^{2}$ for all $a$ ). This problem was first solved in the finite-dimensional case [10], and it was later extended to von Neumann algebras [1]. In the case of the algebra $\mathcal{B}(X)$, A. A. Jafarian and A. R. Sourour established in [7] that every unital surjective linear map $\Phi$ on $\mathcal{B}(X)$, preserving in both directions invertibility, has one of the following two forms

$$
T \mapsto A T A^{-1} \quad \text { or } \quad T \mapsto A T^{*} A^{-1},
$$

where $A$ is a bounded linear operator between suitable spaces. Later, it was shown in [6] that every unital surjective additive preserver of injective operators or of surjective operators in both directions takes one of the two forms (1.1).

Since injective and surjective operators are precisely those operators with zero ascent and descent respectively, the following question arises: What can we say about surjective linear maps on $\mathcal{B}(X)$ preserving in both directions operators of finite ascent and descent, respectively?

Let $H$ be a separable complex infinite-dimensional Hilbert space, and denote by $\mathcal{A}(H)$ (resp. $\mathcal{D}(H))$ the set of all operators in $\mathcal{B}(H)$ of finite ascent (resp. descent). In [11], the authors showed that a surjective additive continuous map $\Phi: \mathcal{B}(H) \rightarrow \mathcal{B}(H)$ preserves $\mathcal{A}(H)$ or $\mathcal{D}(H)$ in both directions if and only if

$$
\Phi(T)=c A T A^{-1} \quad \text { for all } T \in \mathcal{B}(H),
$$

where $c$ is a non-zero scalar and $A: H \rightarrow H$ is an invertible bounded linear, or conjugate linear, operator. An analog result was proved for $\mathcal{A}(H) \cup \mathcal{D}(H)$ by the same authors, see [12]. It should be noted that the question of removing the continuity condition or extending these results to the context of Banach spaces is still open.

The above results motivated us to continue the study of additive preservers involving the ascent and descent. This study may be considered as a key step towards a deeper understanding of operators with finite ascent or descent and their topological properties. In this paper, we will show that if we limit the variation of the ascent and the descent, then we obtain the same conclusion as in [11] without considering continuous preservers.

For each integer $n \geq 1$ let us introduce the following subsets of $\mathcal{B}(H)$ :

1. $\mathcal{A}_{n}(H)$ the set of all operators $T \in \mathcal{B}(H)$ with $\mathrm{a}(T) \leq n$;

2. $\mathcal{D}_{n}(H)$ the set of all operators $T \in \mathcal{B}(H)$ with $\mathrm{d}(T) \leq n$.

Now, we summarize the first main result in the following theorem:

Theorem 1.1. Let $\Phi: \mathcal{B}(H) \rightarrow \mathcal{B}(H)$ be an additive surjective map. Then the following assertions are equivalent:

1. $\Phi$ preserves $\mathcal{A}_{n}(H)$ in both directions;

2. $\Phi$ preserves $\mathcal{D}_{n}(H)$ in both directions; 
3. $\Phi$ preserves $\mathcal{A}_{n}(H) \cup \mathcal{D}_{n}(H)$ in both directions;

4. there exist a non-zero scalar $c$ and a bounded invertible linear, or conjugate linear, operator $A: H \rightarrow H$ such that

$$
\Phi(T)=c A T A^{-1} \quad \text { for all } T \in \mathcal{B}(H) .
$$

Unfortunately, the approach used here does not allow us to obtain an analogue result in the context of Banach spaces. More precisely, one of the most important steps in the proof of the previous theorem consists in determining the topological interior of $\mathcal{A}_{n}(H), \mathcal{D}_{n}(H)$, and $\mathcal{A}_{n}(H) \cup \mathcal{D}_{n}(H)$ using that of $\mathcal{A}(H) \cup \mathcal{D}(H)$, which is known only in the context of separable Hilbert spaces, see [12].

Recall that an operator $T \in \mathcal{B}(X)$ is called upper (resp. lower) semi-Fredholm if $\operatorname{ran}(T)$ is closed and $\operatorname{dim} \operatorname{ker}(T)$ (resp. codim $\operatorname{ran}(T)$ ) is finite. The following properties will be used tacitly throughout the paper (see [15, Section 16]):

1. If the codimension of the range $\operatorname{ran}(T)$ of an operator $T \in \mathcal{B}(X)$ is finite, then $\operatorname{ran}(T)$ is automatically closed;

2. The composition of two upper (resp. lower) semi-Fredholm operators is an upper (resp. lower) semi-Fredholm operator;

3. If $S T$ is an upper (resp. lower) semi-Fredholm operator, then $T$ (resp. $S$ ) is upper (resp. lower) semi-Fredholm.

In [14], the authors studied all linear maps $\Phi$ on $\mathcal{B}(H)$ preserving in both directions semi-Fredholm operators. It has been shown that such maps $\Phi$ preserve in both directions the ideal of compact operators, and that the induced maps on the Calkin algebra are Jordan automorphisms. The problem of determining the structure of such maps on the whole space $\mathcal{B}(H)$ has remained open, and hence they conjectured that $\Phi$ is of the form $T \mapsto A T B+\Psi(T)$ where $A, B \in \mathcal{B}(H)$ are Fredholm operators and $\Psi$ is a linear map on $\mathcal{B}(H)$ whose range is contained in the ideal of compact operators.

In this paper, we prove that if we limit the variation of the ascent (resp. descent) of upper (resp. lower) semi-Fredholm operators, then we obtain the complete description of all additive preservers of such operators in the context of Banach spaces. More precisely, we consider additive preservers of the following subsets of $\mathcal{B}(X)$ :

1. $\mathcal{F}_{n}^{+}(X)$ the set of all upper semi-Fredholm operators $T \in \mathcal{B}(X)$ with $\mathrm{a}(T) \leq n$;

2. $\mathcal{F}_{n}^{-}(X)$ the set of all lower semi-Fredholm operators $T \in \mathcal{B}(X)$ with $\mathrm{d}(T) \leq n$;

3. $\mathcal{F}_{n}^{ \pm}(X)=\mathcal{F}_{n}^{+}(X) \cup \mathcal{F}_{n}^{-}(X)$.

The second main result of the present paper is stated as follows:

Theorem 1.2. Let $\Phi: \mathcal{B}(X) \rightarrow \mathcal{B}(X)$ be an additive surjective map preserving any one of the subsets $\mathcal{F}_{n}^{+}(X), \mathcal{F}_{n}^{-}(X)$ or $\mathcal{F}_{n}^{ \pm}(X)$ in both directions. Then there exist a non-zero scalar $c$, and either a bounded invertible linear, or conjugate linear, operator $A: X \rightarrow X$ such that

$$
\Phi(T)=c A T A^{-1} \quad \text { for all } T \in \mathcal{B}(X),
$$

or, a bounded invertible linear, or conjugate linear, operator $B: X^{*} \rightarrow X$ such that

$$
\Phi(T)=c B T^{*} B^{-1} \quad \text { for all } T \in \mathcal{B}(X) .
$$

As an application of Theorem 1.2, we derive the following corollary: 
Corollary 1.3. Let $\Phi: \mathcal{B}(H) \rightarrow \mathcal{B}(H)$ be an additive surjective map. Then the following assertions are equivalent:

1. $\Phi$ preserves $\mathcal{F}_{n}^{+}(H)$ in both directions;

2. $\Phi$ preserves $\mathcal{F}_{n}^{-}(H)$ in both directions;

3. $\Phi$ preserves $\mathcal{F}_{n}^{ \pm}(H)$ in both directions;

4. there exist a non-zero scalar $c$ and a bounded invertible linear, or conjugate linear, operator $A: H \rightarrow H$ such that

$$
\Phi(T)=c A T A^{-1} \quad \text { for all } T \in \mathcal{B}(H) .
$$

The paper is organized as follows. In the second section, we give the topological interior of each of the subsets $\mathcal{A}_{n}(H), \mathcal{D}_{n}(H)$, and $\mathcal{A}_{n}(H) \cup \mathcal{D}_{n}(H)$. The third section is devoted to establish some useful results on rank-one perturbations of these topological interiors. These results are needed for proving our theorems in the last section.

\section{Topological interior of $\mathcal{A}_{n}(H), \mathcal{D}_{n}(H)$, and $\mathcal{A}_{n}(H) \cup \mathcal{D}_{n}(H)$}

Recall that the hyper-kernel and the hyper-range of an operator $T \in \mathcal{B}(X)$ are respectively the subspaces $\mathcal{N}^{\infty}(T)=\bigcup_{k \geq 0} \operatorname{ker}\left(T^{k}\right)$ and $\mathcal{R}^{\infty}(T)=\bigcap_{k \geq 0} \operatorname{ran}\left(T^{k}\right)$.

Let us introduce the following subsets of $\mathcal{B}(X)$ :

1. $\mathcal{B}_{n}^{+}(X)=\left\{T \in \mathcal{B}(X): \operatorname{ran}(T)\right.$ is closed and $\left.\operatorname{dim} \mathcal{N}^{\infty}(T) \leq n\right\}$;

2. $\mathcal{B}_{n}^{-}(X)=\left\{T \in \mathcal{B}(X): \operatorname{codim} \mathcal{R}^{\infty}(T) \leq n\right\}$;

3. $\mathcal{B}_{n}^{ \pm}(X)=\mathcal{B}_{n}^{+}(X) \cup \mathcal{B}_{n}^{-}(X)$.

One of the most important steps in the proof of our main theorems is to show that the maps we are dealing with preserve the subsets $\mathcal{B}_{n}^{+}(X), \mathcal{B}_{n}^{-}(X)$ and $\mathcal{B}_{n}^{ \pm}(X)$ in both directions. In order to prove this implication, we establish that the topological interior of $\mathcal{A}_{n}(H), \mathcal{D}_{n}(H)$ and $\mathcal{A}_{n}(H) \cup \mathcal{D}_{n}(H)$ is respectively $\mathcal{B}_{n}^{+}(H), \mathcal{B}_{n}^{-}(H)$ and $\mathcal{B}_{n}^{ \pm}(H)$. Similar results are given for $\mathcal{F}_{n}^{+}(X), \mathcal{F}_{n}^{-}(X)$ and $\mathcal{F}_{n}^{ \pm}(X)$.

It should be noted that the ascent and the hyper-kernel of an operator $T \in \mathcal{B}(X)$ are related by the following inequality (see [17])

$$
\mathrm{a}(T) \leq \operatorname{dim} \mathcal{N}^{\infty}(T)
$$

Similarly, the descent is related to the hyper-range by

$$
\mathrm{d}(T) \leq \operatorname{codim} \mathcal{R}^{\infty}(T) .
$$

Remark 2.1. For $T \in \mathcal{B}(X)$, it follows easily from the definition of the ascent and of the descent that:

1. $\operatorname{dim} \operatorname{ker}\left(T^{n+1}\right) \leq n$ if and only if $\operatorname{dim} \mathcal{N}^{\infty}(T) \leq n$;

2. codim $\operatorname{ran}\left(T^{n+1}\right) \leq n$ if and only if $\operatorname{codim} \mathcal{R}^{\infty}(T) \leq n$.

Proposition 2.2. $\mathcal{B}_{n}^{+}(X), \mathcal{B}_{n}^{-}(X)$ and $\mathcal{B}_{n}^{ \pm}(X)$ are open subsets of $\mathcal{F}_{n}^{+}(X), \mathcal{F}_{n}^{-}(X)$ and $\mathcal{F}_{n}^{ \pm}(X)$, respectively. 
Proof. It follows from $(2.1)$ and $(2.2)$ that $\mathcal{B}_{n}^{+}(X)$ and $\mathcal{B}_{n}^{-}(X)$ are subsets of $\mathcal{F}_{n}^{+}(X)$ and $\mathcal{F}_{n}^{-}(X)$ respectively, and so $\mathcal{B}_{n}^{ \pm}(X)$ is a subset of $\mathcal{F}_{n}^{ \pm}(X)$. Let $S \in \mathcal{B}_{n}^{+}(X)$. In particular, we have $\operatorname{dim} \operatorname{ker}\left(S^{n}\right)=\operatorname{dim} \operatorname{ker}\left(S^{n+1}\right) \leq n$ and $S^{n+1}$ is an upper semiFredholm operator. Hence, it follows by [15, Theorem 16.11] that there exists $\eta>0$ such that for $T \in \mathcal{B}(X)$ with $\left\|T-S^{n+1}\right\|<\eta$, we have that $T$ is upper semi-Fredholm and

$$
\operatorname{dim} \operatorname{ker}(T) \leq \operatorname{dim} \operatorname{ker}\left(S^{n+1}\right) \leq n .
$$

On the other hand, since the function $T \mapsto T^{n+1}$ is continuous on $\mathcal{B}(X)$, there exists $\varepsilon>0$ such that

$$
\left\|T^{n+1}-S^{n+1}\right\|<\eta \quad \text { for all } T \in \mathcal{B}(X) \text { with }\|T-S\|<\varepsilon .
$$

Combining (2.4) and (2.3) we obtain that $T^{n+1}$ is upper semi-Fredholm and

$$
\operatorname{dim} \operatorname{ker}\left(T^{n+1}\right) \leq \operatorname{dim} \operatorname{ker}\left(S^{n+1}\right) \leq n,
$$

and so $T \in \mathcal{B}_{n}^{+}(X)$ for all $T \in \mathcal{B}(X)$ with $\|T-S\|<\varepsilon$. This shows that $\mathcal{B}_{n}^{+}(X)$ is open.

Similarly, we prove that $\mathcal{B}_{n}^{-}(X)$ is open, and hence $\mathcal{B}_{n}^{ \pm}(X)$ is also open.

From [5, Lemma 1.1], given a non-negative integer $d$, we have

$$
\mathrm{a}(T) \leq d \Leftrightarrow \operatorname{ker}\left(T^{m}\right) \cap \operatorname{ran}\left(T^{d}\right)=\{0\} \text { for some } m \geq 1 .
$$

Remark 2.3. Let $T \in \mathcal{B}(X)$. Then the following assertions hold:

1. If $T$ has finite ascent and descent then $\mathrm{a}(T)=\mathrm{d}(T)$ and $X=\operatorname{ker}\left(T^{k}\right) \oplus \operatorname{ran}\left(T^{k}\right)$, where $k=\mathrm{a}(T)$ and the direct sum is topological (see [15, Corollary 20.5]).

2. If $T=T_{1} \oplus T_{2}$ with respect to any decomposition of $X$, then it follows from [18, Theorem 6.1] that

$$
\mathrm{a}(T)=\max \left\{\mathrm{a}\left(T_{1}\right), \mathrm{a}\left(T_{2}\right)\right\} \quad \text { and } \quad \mathrm{d}(T)=\max \left\{\mathrm{d}\left(T_{1}\right), \mathrm{d}\left(T_{2}\right)\right\} .
$$

The following example shows that $\mathcal{B}_{n}^{+}(X), \mathcal{B}_{n}^{-}(X)$ and $\mathcal{B}_{n}^{ \pm}(X)$ are proper subsets of $\mathcal{F}_{n}^{+}(X), \mathcal{F}_{n}^{-}(X)$ and $\mathcal{F}_{n}^{ \pm}(X)$, respectively, and that there exist operators with finite ascent and descent which are not semi-Fredholm.

Example 2.4. Let $Y \subset X$ be a closed subspace of dimension $n+1$, and write $X=Y \oplus Z$ where $Z$ is a closed subspace of $X$. With respect to this decomposition, consider the operator $T=0 \oplus I$. According to the previous remark, one can easily see that $\mathrm{a}(T)=\mathrm{d}(T)=1$. Since $\mathcal{N}^{\infty}(T)=\operatorname{ker}(T)=Y$ and $\mathcal{R}^{\infty}(T)=\operatorname{ran}(T)=Z$, then $T$ belongs to $\mathcal{F}_{n}^{+}(X) \cap \mathcal{F}_{n}^{-}(X)$ and not to $\mathcal{B}_{n}^{ \pm}(X)$.

Similarly, for $S=I-T$, we have a $(S)=\mathrm{d}(S)=1, \operatorname{ker}(S)=Z$ and $\operatorname{ran}(S)=Y$. Thus, $S$ is not a semi-Fredholm operator.

Recall that an operator $T \in \mathcal{B}(X)$ is called upper (resp. lower) semi-Browder if it is upper (resp. lower) semi-Fredholm of finite ascent (resp. descent). Clearly, every operator in $\mathcal{F}_{n}^{+}(X)$ (resp. $\mathcal{F}_{n}^{-}(X)$ ) is upper (resp. lower) semi-Browder.

Theorem 2.5. Let $T \in \mathcal{B}(X)$ be non-zero. The following assertions are equivalent:

1. $T \in \mathcal{B}_{n}^{ \pm}(X)\left(\right.$ resp. $\left.\mathcal{B}_{n}^{+}(X), \mathcal{B}_{n}^{-}(X)\right)$; 
2. for every $S \in \mathcal{B}(X)$ there exists $\varepsilon_{0}>0$ such that $T+\varepsilon S \in \mathcal{F}_{n}^{ \pm}(X)$ (resp. $\mathcal{F}_{n}^{+}(X)$, $\left.\mathcal{F}_{n}^{-}(X)\right)$, for all numbers (equivalently, rational numbers) $|\varepsilon|<\varepsilon_{0}$.

Proof. (1) $\Rightarrow(2)$ follows immediately from the previous proposition.

$(2) \Rightarrow(1)$. Suppose that for every $S \in \mathcal{B}(X)$ there exists $\varepsilon_{0}>0$ such that $T+\varepsilon S \in \mathcal{F}_{n}^{ \pm}(X)$ for all numbers $|\varepsilon|<\varepsilon_{0}$. In particular, we have $T \in \mathcal{F}_{n}^{ \pm}(X)$, and so $T$ is either upper semi-Browder or lower semi-Browder. It follows from $[15$, Theorem 20.10] that there exist two closed $T$-invariant subspaces $X_{1}$ and $X_{2}$ such that $X=X_{1} \oplus X_{2}, \operatorname{dim} X_{1}<\infty, T_{1}=T_{\mid X_{1}}$ is nilpotent and $T_{\mid X_{2}}$ is either bounded below or onto, respectively. We claim that $\operatorname{dim} X_{1} \leq n$. Let $\left\{e_{i}: 0 \leq i \leq p\right\}$ be a basis of $X_{1}$ such that $T e_{0}=0$ and $T e_{i}=\varepsilon_{i} e_{i-1}$ for $1 \leq i \leq p$ where $\varepsilon_{i} \in\{0,1\}$. With respect to the decomposition of $X$, consider the operator $S \in \mathcal{B}(X)$ given by $S=S_{1} \oplus 0$ where $S_{1} e_{0}=0$ and $S_{1} e_{i}=e_{i-1}$ for $1 \leq i \leq p$. Clearly, for $\varepsilon \notin\{-1,0\}$ we have

$$
\left(T_{1}+\varepsilon S_{1}\right) e_{0}=0 \quad \text { and } \quad\left(T_{1}+\varepsilon S_{1}\right) e_{i}=\left(\varepsilon_{i}+\varepsilon\right) e_{i-1} \text { for } 1 \leq i \leq p .
$$

Hence $\left(T_{1}+\varepsilon S_{1}\right)^{p} e_{p}=\lambda e_{0} \neq 0$ where $\lambda=\left(\varepsilon_{p}+\varepsilon\right) \ldots\left(\varepsilon_{1}+\varepsilon\right)$.

Therefore $e_{0} \in \operatorname{ker}\left(T_{1}+\varepsilon S_{1}\right) \cap \operatorname{ran}\left(T_{1}+\varepsilon S_{1}\right)^{p}$, and consequently

$$
\mathrm{a}\left(T_{1}+\varepsilon S_{1}\right)=\mathrm{d}\left(T_{1}+\varepsilon S_{1}\right) \geq p+1
$$

by (2.5). But, we have also

$$
\mathrm{a}\left(T_{1}+\varepsilon S_{1}\right) \leq \mathrm{a}(T+\varepsilon S) \quad \text { and } \quad \mathrm{d}\left(T_{1}+\varepsilon S_{1}\right) \leq \mathrm{d}(T+\varepsilon S) .
$$

Since $T+\varepsilon S \in \mathcal{F}_{n}^{ \pm}(X)$, then $\mathrm{a}(T+\varepsilon S) \leq n$ or $\mathrm{d}(T+\varepsilon S) \leq n$. Thus $\operatorname{dim} X_{1} \leq n$.

Now, if $T \in \mathcal{F}_{n}^{+}(X)$ (resp. $\mathcal{F}_{n}^{-}(X)$ ) then $T$ is upper (resp. lower) semi-Browder, and so the space $X_{1}$ (resp. $\left.X_{2}\right)$ is uniquely determined and $X_{1}=\mathcal{N}^{\infty}(T)$ (resp. $X_{2}=$ $\mathcal{R}^{\infty}(T)$ ) (see $\left[15\right.$, Theorem 20.10]). This proves that $T \in \mathcal{B}_{n}^{+}(X)$ (resp. $\left.\mathcal{B}_{n}^{-}(X)\right)$.

For a subset $\Gamma \subseteq \mathcal{B}(X)$, we write $\operatorname{Int}(\Gamma)$ for its interior. As a consequence of Theorem 2.5 , we derive the following corollary.

Corollary 2.6. We have $\operatorname{Int}\left(\mathcal{F}_{n}^{+}(X)\right)=\mathcal{B}_{n}^{+}(X), \operatorname{Int}\left(\mathcal{F}_{n}^{-}(X)\right)=\mathcal{B}_{n}^{-}(X)$ and $\operatorname{Int}\left(\mathcal{F}_{n}^{ \pm}(X)\right)=\mathcal{B}_{n}^{ \pm}(X)$.

Proof. Let us show that $\operatorname{Int}\left(\mathcal{F}_{n}^{+}(X)\right)=\mathcal{B}_{n}^{+}(X)$. Note that $\mathcal{B}_{n}^{+}(X) \subseteq \operatorname{Int}\left(\mathcal{F}_{n}^{+}(X)\right)$ because $\mathcal{B}_{n}^{+}(X)$ is open. Let $T \notin \mathcal{B}_{n}^{+}(X)$, then Theorem 2.5 ensures the existence of an operator $S \in \mathcal{B}(X)$ and a sequence $\left(\varepsilon_{k}\right)$ converging to zero such that $T+\varepsilon_{k} S \notin \mathcal{F}_{n}^{+}(X)$ for all $k \geq 0$. Consequently, $T \notin \operatorname{Int}\left(\mathcal{F}_{n}^{+}(X)\right)$.

Similarly, we prove that $\operatorname{Int}\left(\mathcal{F}_{n}^{-}(X)\right)=\mathcal{B}_{n}^{-}(X)$ and $\operatorname{Int}\left(\mathcal{F}_{n}^{ \pm}(X)\right)=\mathcal{B}_{n}^{ \pm}(X)$.

Theorem 2.7. Let $H$ be a separable complex infinite-dimensional Hilbert space and let $T \in \mathcal{B}(H)$. Then the following assertions are equivalent:

1. $T \in \mathcal{B}_{n}^{ \pm}(H)\left(\operatorname{resp} . \mathcal{B}_{n}^{+}(H), \mathcal{B}_{n}^{-}(H)\right)$;

2. for every $S \in \mathcal{B}(H)$ there exists $\varepsilon_{0}>0$ such that $T+\varepsilon S \in \mathcal{A}_{n}(H) \cup \mathcal{D}_{n}(H)$ (resp. $\mathcal{A}_{n}(H), \mathcal{D}_{n}(H)$ ), for all numbers (equivalently, rational numbers) $|\varepsilon|<\varepsilon_{0}$. 
Proof. $(1) \Rightarrow(2)$ follows immediately from Proposition 2.2.

$(2) \Rightarrow(1)$. Suppose that for every $S \in \mathcal{B}(H)$ there exists $\varepsilon_{0}>0$ such that $T+\varepsilon S \in \mathcal{A}_{n}(H) \cup \mathcal{D}_{n}(H)$ for all $|\varepsilon|<\varepsilon_{0}$. Then, using [12, Proposition 2.5], we get that $T$ is a semi-Browder operator. The rest of the proof is similar to the proof of Theorem 2.5.

Using a similar proof of Corollary 2.6, we get the following result.

Corollary 2.8. We have $\operatorname{Int}\left(\mathcal{A}_{n}(H) \cup \mathcal{D}_{n}(H)\right)=\mathcal{B}_{n}^{ \pm}(H), \operatorname{Int}\left(\mathcal{A}_{n}(H)\right)=\mathcal{B}_{n}^{+}(H)$ and $\operatorname{Int}\left(\mathcal{D}_{n}(H)\right)=\mathcal{B}_{n}^{-}(H)$.

\section{3. $\mathcal{B}_{n}^{+}(X), \mathcal{B}_{n}^{-}(X)$ and $\mathcal{B}_{n}^{ \pm}(X)$ under rank-one perturbations}

Let $z \in X$ and let $f \in X^{*}$ be non-zero. We denote by $z \otimes f$ the rank-one operator defined by $(z \otimes f)(x)=f(x) z$ for all $x \in X$. Note that every rank-one operator in $\mathcal{B}(X)$ can be written in this form.

In [13], the authors proved that for a rank-one operator $F \in \mathcal{B}(X)$ and for $T \in$ $\mathcal{B}(X)$ with $\operatorname{dim} \operatorname{ker}(T) \leq n$, we have either $\operatorname{dim} \operatorname{ker}(T+F) \leq n$ or $\operatorname{dim} \operatorname{ker}(T-F) \leq n$. In the following, we extend this result to the setting of the hyper-kernel subspace.

Proposition 3.1. Let $T \in \mathcal{B}(X)$ be such that $\operatorname{dim} \mathcal{N}^{\infty}(T) \leq n$, and let $F \in \mathcal{B}(X)$ be a rank-one operator. Then either $\operatorname{dim} \mathcal{N}^{\infty}(T+F) \leq n$ or $\operatorname{dim} \mathcal{N}^{\infty}(T-F) \leq n$.

Before giving the proof of this proposition, we need to establish some lemmas. For $T, F \in \mathcal{B}(X)$, let

$$
\mathrm{M}(T, F)=\left\{x \in \mathcal{N}^{\infty}(T): F T^{i} x=0 \text { for all } i \geq 0\right\} .
$$

Clearly, $\mathrm{M}(T, F)$ is a $T$-invariant subspace of $\mathcal{N}^{\infty}(T) \cap \operatorname{ker}(F)$. Furthermore, if $T$ has a finite ascent, then $\mathrm{M}(T, F)$ is closed.

Lemma 3.2. Let $T \in \mathcal{B}(X)$ be non-zero, and let $F=z \otimes f$ be a rank-one operator such that $\operatorname{ker}(T) \cap \operatorname{ker}(F)=\{0\}$. Assume that there exist an integer $m \geq 0$ and a vector $x \in \operatorname{ker}(T+F)^{m+1} \backslash \operatorname{ker}(T+F)^{m}$ such that $x \notin \mathrm{M}(T, F)$. Then $x$ is a linear combination of linearly independent vectors $x_{i}, 0 \leq i \leq m$, such that

$$
(T+F) x_{0}=0,(T+F) x_{i}=x_{i-1} \text { for } 1 \leq i \leq m \text {, and } f\left(x_{i}\right)=\delta_{i 0} \text { for } 0 \leq i \leq m .
$$

Proof. Let $u_{i}=(T+F)^{m-i} x$ for $0 \leq i \leq m$. It follows that $u_{i}, 0 \leq i \leq m$, are linearly independent vectors, $(T+F) u_{0}=0$ and $(T+F) u_{i}=u_{i-1}$ for $1 \leq i \leq m$. Since $\operatorname{ker}(T) \cap \operatorname{ker}(F)=\{0\}$, we infer that $f\left(u_{0}\right) \neq 0$. Without loss of generality we may assume that $f\left(u_{0}\right)=1$. Consider the scalars $c_{0}, c_{1}, \ldots, c_{m-1}$ defined inductively by

$$
\begin{aligned}
& c_{0}=-f\left(u_{1}\right) \\
& c_{1}=-c_{0} f\left(u_{1}\right)-f\left(u_{2}\right) \\
& c_{2}=-c_{1} f\left(u_{1}\right)-c_{0} f\left(u_{2}\right)-f\left(u_{3}\right) \\
& \vdots \\
& c_{m-1}=-c_{m-2} f\left(u_{1}\right)-\cdots-c_{0} f\left(u_{m-1}\right)-f\left(u_{m}\right) .
\end{aligned}
$$


This means that we have

$$
f\left(u_{i}\right)+\sum_{k=1}^{i} c_{i-k} f\left(u_{k-1}\right)=0 \text { for } 1 \leq i \leq m .
$$

Let $x_{0}=u_{0}$ and $x_{i}=u_{i}+\sum_{k=1}^{i} c_{i-k} u_{k-1}$ for $1 \leq i \leq m$. Clearly, the vectors $x_{i}$, $0 \leq i \leq m$, are linearly independent. Moreover, it follows from (3.1) that $f\left(x_{i}\right)=\delta_{i 0}$ for $0 \leq i \leq m$. Furthermore, we have $(T+F) x_{0}=(T+F) u_{0}=0$ and

$$
(T+F) x_{i}=(T+F) u_{i}+\sum_{k=1}^{i} c_{i-k}(T+F) u_{k-1}=u_{i-1}+\sum_{k=2}^{i} c_{i-k} u_{k-2}=x_{i-1}
$$

for $1 \leq i \leq m$. Finally, we have

$$
x=u_{m} \in \operatorname{Span}\left\{u_{i}: 0 \leq i \leq m\right\}=\operatorname{Span}\left\{x_{i}: 0 \leq i \leq m\right\} .
$$

This completes the proof.

The following lemma is a special case of Proposition 3.1, and it will be required for proving that proposition.

Lemma 3.3. Let $T \in \mathcal{B}(X)$ be such that $\operatorname{dim} \mathcal{N}^{\infty}(T) \leq n$, and let $F \in \mathcal{B}(X)$ be a rank-one operator such that $\operatorname{ker}(T) \cap \operatorname{ker}(F)=\{0\}$. Then either $\operatorname{dim} \mathcal{N}^{\infty}(T+F) \leq n$ or $\operatorname{dim} \mathcal{N}^{\infty}(T-F) \leq n$.

Proof. Write $F=z \otimes f$ where $z \in X$ and $f \in X^{*}$ are non-zero. Clearly, if either $\operatorname{ker}(T+F)^{n+1}$ or $\operatorname{ker}(T-F)^{n+1}$ is contained in $\mathrm{M}(T, F)$, then either $\operatorname{dim} \mathcal{N}^{\infty}(T+F) \leq$ $n$ or $\operatorname{dim} \mathcal{N}^{\infty}(T-F) \leq n$ respectively. Hence, we may assume that $\operatorname{ker}(T+F)^{n+1} \nsubseteq$ $\mathrm{M}(T, F)$ and $\operatorname{ker}(T-F)^{n+1} \nsubseteq \subseteq \mathrm{M}(T, F)$. Let $0 \leq m, p \leq n$ be the biggest integers for which there exist $x \in \operatorname{ker}(T+F)^{m+1} \backslash \operatorname{ker}(T+F)^{m}$ and $y \in \operatorname{ker}(T-F)^{p+1} \backslash \operatorname{ker}(T-F)^{p}$ such that $x, y \notin \mathrm{M}(T, F)$. Without loss of generality we can assume that $m \leq p$. We will show that $\operatorname{dim} \mathcal{N}^{\infty}(T+F) \leq n$. Using the previous lemma, we infer that $y$ is a linear combination of linearly independent vectors $y_{i}, 0 \leq i \leq p$, such that

$$
(T-F) y_{0}=0,(T-F) y_{i}=y_{i-1} \text { for } 1 \leq i \leq p, \text { and } f\left(y_{i}\right)=\delta_{i 0} \text { for } 0 \leq i \leq p .
$$

From this, one can easily see that $(T+F) y_{0}=2 z$ and $(T+F) y_{i}=T y_{i}=y_{i-1}$ for $1 \leq i \leq p$, and so $(T+F)^{k} y_{i}=y_{i-k}$ for $0 \leq k \leq i \leq p$. Thus, we get easily that

$$
I+\sum_{i=0}^{p} y_{i} \otimes f(T+F)^{i}=\prod_{i=0}^{p}\left(I+y_{i} \otimes f(T+F)^{i}\right) .
$$

Furthermore, since $f\left((T+F)^{i} y_{i}\right)=f\left(y_{0}\right)=1$ for $0 \leq i \leq p$, the above equation defines an invertible operator denoted by $S$.

Let $u \in \operatorname{ker}(T+F)^{n+1}$ be an arbitrary non-zero vector, and let $0 \leq r \leq n$ be such that $u \in \operatorname{ker}(T+F)^{r+1} \backslash \operatorname{ker}(T+F)^{r}$. If $u \in \mathrm{M}(T, F)$, then $f\left(T^{i} u\right)=0$, and so $(T+F)^{i} u=T^{i} u$ for every $i \geq 0$. Hence, $S u=u \in \mathrm{M}(T, F) \subseteq \mathcal{N}^{\infty}(T)$. Consider the 
case when $u \notin \mathrm{M}(T, F)$. Then, Lemma 3.2 asserts that $u$ is a linear combination of linearly independent vectors $x_{i}, 0 \leq i \leq r$, satisfying

$$
(T+F) x_{0}=0,(T+F) x_{i}=x_{i-1} \text { for } 1 \leq i \leq r \text {, and } f\left(x_{i}\right)=\delta_{i 0} \text { for } 0 \leq i \leq r .
$$

It follows that $(T+F)^{k} x_{i}=x_{i-k}$ for $k \geq 0$ and $0 \leq i \leq r$, where we set formally $x_{j}=0$ for $j<0$. Now, by the definition of $m$, we have $r \leq m \leq p$. This allows us to obtain easily that $S x_{i}=x_{i}+y_{i}$ for $0 \leq i \leq r$. It follows that $T^{i} S x_{i}=$ $x_{0}+y_{0} \in \operatorname{ker}(T)$, and hence $S x_{i} \in \mathcal{N}^{\infty}(T)$ for $0 \leq i \leq r$. Consequently, we get that $S u \in \mathcal{N}^{\infty}(T)$. The vector $u$ was arbitrary, therefore $\bar{S} \operatorname{ker}(T+F)^{n+1} \subseteq \mathcal{N}^{\infty}(T)$. So that $\operatorname{dim} \operatorname{ker}(T+F)^{n+1} \leq n$. According to Remark 2.1, this completes the proof.

For $T, F \in \mathcal{B}(X)$, we denote respectively by $\tilde{T}$ and $\tilde{F}$ the operators induced by $T$ and $F$ on $X / \mathrm{M}(T, F)$. Note that the hyper-kernels of $\tilde{T}+c \tilde{F}$ and $T+c F$ are related by the following relation (see [17, Lemma 2.9])

$$
\mathcal{N}^{\infty}(\tilde{T}+c \tilde{F})=\mathcal{N}^{\infty}(T+c F) / \mathrm{M}(T, F) \text { for all } c \in \mathbb{K} .
$$

Proof of Proposition 3.1. Firstly, if $\tilde{F}=0$, then it follows from (3.2) that

$$
\mathcal{N}^{\infty}(\tilde{T}+\tilde{F})=\mathcal{N}^{\infty}(T+F) / \mathrm{M}(T, F)=\mathcal{N}^{\infty}(\tilde{T})=\mathcal{N}^{\infty}(T) / \mathrm{M}(T, F) .
$$

So that $\operatorname{dim} \mathcal{N}^{\infty}(T+F)=\operatorname{dim} \mathcal{N}^{\infty}(T) \leq n$.

Now, consider the case $\tilde{F} \neq 0$. Then $z \notin \mathrm{M}(T, F)$, and for every $x \in X$, we have

$$
\begin{aligned}
x+\mathrm{M}(T, F) \in \operatorname{ker}(\tilde{T}) \cap \operatorname{ker}(\tilde{F}) & \Leftrightarrow T x \in \mathrm{M}(T, F) \text { and } F x=f(x) z \in \mathrm{M}(T, F) \\
& \Leftrightarrow T x \in \mathrm{M}(T, F) \text { and } f(x)=0 \\
& \Leftrightarrow x \in \mathrm{M}(T, F) .
\end{aligned}
$$

This implies that $\operatorname{ker}(\tilde{T}) \cap \operatorname{ker}(\tilde{F})=\{0\}$.

Since $\operatorname{dim} \mathcal{N}^{\infty}(\tilde{T}) \leq n-q$ where $q=\operatorname{dim} \mathrm{M}(T, F)$, the previous lemma ensures that either $\operatorname{dim} \mathcal{N}^{\infty}(\tilde{T}+\tilde{F}) \leq n-q$ or $\operatorname{dim} \mathcal{N}^{\infty}(\tilde{T}-\tilde{F}) \leq n-q$. Thus, we get that either $\operatorname{dim} \mathcal{N}^{\infty}(T+F) \leq n$ or $\operatorname{dim} \mathcal{N}^{\infty}(T-F) \leq n$. This completes the proof.

Throughout the sequel, $\Lambda$ will denote any of the subsets $\mathcal{B}_{n}^{+}(X), \mathcal{B}_{n}^{-}(X)$ or $\mathcal{B}_{n}^{ \pm}(X)$. Also, the subset $\mathcal{B}_{n}(X)=\mathcal{B}_{n}^{+}(X) \cap \mathcal{B}_{n}^{-}(X)$, introduced and studied in [17], will be used in the rest of this paper.

Recall that for a semi-Fredholm operator $T \in \mathcal{B}(X)$, the index is defined by

$$
\operatorname{ind}(T)=\operatorname{dim} \operatorname{ker}(T)-\operatorname{codim} \operatorname{ran}(T),
$$

and if the index is finite, $T$ is said to be Fredholm. It should be noted that if ind $(T)=0$ then $\mathrm{a}(T)=\mathrm{d}(T)$ (see [12, Lemma 2.3]). Moreover, in this case

$$
T \in \Lambda \Leftrightarrow T \in \mathcal{B}_{n}(X) \Leftrightarrow \operatorname{dim} \mathcal{N}^{\infty}(T) \leq n .
$$

Proposition 3.4. Let $T \in \Lambda$ and let $F \in \mathcal{B}(X)$ be a rank-one operator. Then either $T+F \in \Lambda$ or $T-F \in \Lambda$.

Before proving this proposition, a duality relation between $\mathcal{B}_{n}^{+}(X)$ and $\mathcal{B}_{n}^{-}(X)$ should be established first. For a subset $M \subseteq X$, we denote by $M^{\perp}=\left\{f \in X^{*}: M \subseteq \operatorname{ker}(f)\right\}$ its annihilator. 
Lemma 3.5. Let $T$ be a bounded operator on $X$. Then :

$$
\left.T \in \mathcal{B}_{n}^{+}(X)\left(\text { resp. } \mathcal{B}_{n}^{-}(X)\right) \quad \Leftrightarrow \quad T^{*} \in \mathcal{B}_{n}^{-}\left(X^{*}\right) \text { (resp. } \mathcal{B}_{n}^{+}\left(X^{*}\right)\right) .
$$

Proof. Suppose that $T \in \mathcal{B}_{n}^{+}(X)$. In particular, $T$ is a semi-Fredholm operator, and so $\operatorname{ran}\left(T^{k}\right)$ is closed for every $k \geq 0$. Since a $(T) \leq n$, it follows from [15, Corollary A.1.17] that

$$
\operatorname{ker}\left(T^{n+1}\right)^{\perp}=\operatorname{ker}\left(T^{n}\right)^{\perp}=\operatorname{ran}\left(\left(T^{*}\right)^{n+1}\right)=\operatorname{ran}\left(\left(T^{*}\right)^{n}\right) .
$$

Thus, $\mathrm{d}\left(T^{*}\right) \leq n$. Using [15, Theorem A.1.20] we get that $\operatorname{codim} \mathcal{R}^{\infty}\left(T^{*}\right)=\operatorname{codim} \operatorname{ran}\left(\left(T^{*}\right)^{n}\right)=\operatorname{dim} \operatorname{ker}\left(T^{n}\right)=\operatorname{dim} \mathcal{N}^{\infty}(T) \leq n$.

So that $T^{*} \in \mathcal{B}_{n}^{-}\left(X^{*}\right)$. The proofs of the converse and of the statement for $\mathcal{B}_{n}^{-}(X)$ are similar.

Proof of Proposition 3.4. Let $T \in \Lambda$, and let $F \in \mathcal{B}(X)$ be a rank-one operator. It follows from [15, Theorem 16.16] that $T+F$ and $T-F$ are semi-Fredholm. If $T \in \mathcal{B}_{n}^{+}(X)$ then Proposition 3.1 implies that either $T+F \in \mathcal{B}_{n}^{+}(X)$ or $T-F \in \mathcal{B}_{n}^{+}(X)$.

The case when $T \in \mathcal{B}_{n}^{-}(X)$ follows from the first one by duality.

The following theorem, will play a crucial role in proving the main results.

Theorem 3.6. Let $F \in \mathcal{B}(X)$ be a non-zero operator. Then the following assertions hold:

1. There exists an invertible operator $T \in \mathcal{B}(X)$ such that $T+F \notin \Lambda$.

2. If $\operatorname{dim} \operatorname{ran}(F) \geq 2$, then there exists an invertible operator $T \in \mathcal{B}(X)$ such that $T+F \notin \Lambda$ and $T-F \notin \Lambda$.

Proof. Suppose first that $\operatorname{ran}(F)$ has an infinite dimension. Then codim $\operatorname{ker}(F)=\infty$, and hence there exist linearly independent vectors $x_{i}, 0 \leq i \leq 2 n+1$, that generate a subspace having trivial intersection with $\operatorname{ker}(F)$. It follows that the vectors $F x_{i}$, $0 \leq i \leq 2 n+1$, are linearly independent. Write

$$
X=\operatorname{Span}\left\{x_{i}: 0 \leq i \leq 2 n+1\right\} \oplus Y=\operatorname{Span}\left\{F x_{i}: 0 \leq i \leq 2 n+1\right\} \oplus Z,
$$

where $Y, Z$ are two closed subspaces and $Y=F^{-1} Z$. Then there exists an invertible operator $T \in \mathcal{B}(X)$ such that $T Y=Z$, and $T x_{i}=(-1)^{i} F x_{i}$ for $0 \leq i \leq 2 n+1$.

Clearly, $x_{2 i+1} \in \operatorname{ker}(T+F)$ and $x_{2 i} \in \operatorname{ker}(T-F)$ for $0 \leq i \leq n$, and hence

$$
\operatorname{dim} \operatorname{ker}(T \pm F)>n \text {. }
$$

But, we have also

$$
\operatorname{ran}(T+F) \subseteq \operatorname{Span}\left\{F x_{2 i}: 0 \leq i \leq n\right\} \oplus Z
$$

and

$$
\operatorname{ran}(T-F) \subseteq \operatorname{Span}\left\{F x_{2 i+1}: 0 \leq i \leq n\right\} \oplus Z .
$$

Then $\operatorname{codim} \operatorname{ran}(T \pm F)>n$, and so $T \pm F \notin \Lambda$. This establishes the assertions (1) and (2).

Assume now that $F$ is finite-rank, and let $p=\min \{\operatorname{dim} \operatorname{ran}(F), 2\}$. It follows from [17, Proposition 2.12] that there exists an invertible operator $T \in \mathcal{B}(X)$ such that $T+F \notin \mathcal{B}_{n}(X)$ and $T-(-1)^{p} F \notin \mathcal{B}_{n}(X)$. But, $T+F$ and $T-(-1)^{p} F$ are 
Fredholm operators of index zero, then $T+F \notin \Lambda$ and $T-(-1)^{p} F \notin \Lambda$. This completes the proof.

\section{Proofs of the main results}

As a consequence of Theorem 3.6 and Proposition 3.4, we have the following result.

Lemma 4.1. Let $\Phi: \mathcal{B}(X) \rightarrow \mathcal{B}(X)$ be an additive surjective map. If $\Phi$ preserves $\Lambda$ in both directions, then $\Phi$ is injective and it preserves the set of rank-one operators in both directions.

Proof. Suppose on the contrary that there exists $F \neq 0$ such that $\Phi(F)=0$. Then, by Theorem 3.6, there exists an invertible operator $T \in \mathcal{B}(X)$ satisfying $T+F \notin \Lambda$. But, $\Phi(T+F)=\Phi(T) \in \Lambda$. This contradiction proves that $\Phi$ is injective.

Let $F \in \mathcal{B}(X)$ with $\operatorname{dim} \operatorname{ran}(F) \geq 2$. Then it follows again by Theorem 3.6 that there exists an invertible operator $T \in \mathcal{B}(X)$ such that $T+F$ and $T-F$ do not belong to $\Lambda$, and hence $\Phi(T+F)$ and $\Phi(T-F)$ do neither. Therefore, by Proposition 3.4, we obtain that $\operatorname{dim} \operatorname{ran}(\Phi(F)) \geq 2$. Since $\Phi$ is bijective and $\Phi^{-1}$ satisfies the same properties as $\Phi$, we obtain that $\Phi$ preserves the set of rank-one operators in both directions.

Recall that an operator $T \in \mathcal{B}(X)$ is said to be algebraic if there exists a non-zero complex polynomial $P$ for which $P(T)=0$. Such an operator $T$ has finite ascent and descent (see [3, Theorem 2.7] and [4, Theorem 1.5]). Moreover, we have

$$
T \in \Lambda \Leftrightarrow T \in \mathcal{B}_{n}(X) \Leftrightarrow \operatorname{dim} \mathcal{N}^{\infty}(T) \leq n .
$$

Lemma 4.2. Let $\Phi: \mathcal{B}(X) \rightarrow \mathcal{B}(X)$ be an additive surjective map preserving $\Lambda$ in both directions. Then $\Phi(I)=c I$ where $c$ is a non-zero scalar.

Proof. We claim first that $S=\Phi(I)$ is an algebraic operator. Let $x \in X$ be non-zero. If the set $\left\{S^{i} x: 0 \leq i \leq 2 n+1\right\}$ is linearly independent, then there exists a linear form $f \in X^{*}$ such that $f\left(S^{i} x\right)=-\delta_{i, 2 n+1}$ for $0 \leq i \leq 2 n+1$. Let $T=S+S^{n+1} x \otimes f S^{n+1}$. It follows that

$$
T\left(S^{i} x\right)=S^{i+1} x, \text { for } 0 \leq i \leq n-1 \text {, and } T\left(S^{n} x\right)=0 .
$$

Hence $\mathrm{a}(T) \geq n+1$. On the other hand, we have

$$
T^{*}\left(f S^{i}\right)=f S^{i+1}, \text { for } 0 \leq i \leq n-1 \text {, and } T^{*}\left(f S^{n}\right)=0 .
$$

Then $\mathrm{a}\left(T^{*}\right) \geq n+1$, and so $\mathrm{d}(T) \geq n+1$. Thus $T \notin \Lambda$. This contradiction shows that $\left\{S^{i} x: 0 \leq i \leq 2 n+1\right\}$ is a linearly dependent set. The vector $x$ was arbitrary, therefore it follows from [2, Theorem 4.2.7] that $S$ is algebraic.

Now assume, on the contrary, that $S$ is not a scalar multiple of the identity. Then there exists $y_{1} \in X$ such that the vectors $y_{1}$ and $S y_{1}$ are linearly independent. Since $S \in \Lambda$, the subspace $\operatorname{ran}(S)$ has an infinite dimension, and hence there exists 
$y_{i} \in X, 2 \leq i \leq n$, such that $\left\{y_{1}, S y_{i}: 1 \leq i \leq n\right\}$ is a linearly independent set. Consider linear forms $g_{i} \in X^{*}$ such that

$$
g_{i}\left(y_{1}\right)=0 \text { and } g_{i}\left(S y_{j}\right)=-\delta_{i j} \text { for } 1 \leq i, j \leq n .
$$

If we let $F=\sum_{i=1}^{n} S^{2} y_{i} \otimes g_{i}$, we obtain easily that $S y_{j} \in \operatorname{ker}(S+F)$, for $1 \leq j \leq n$, and $(S+F) y_{1}=S y_{1} \in \operatorname{ker}(S+F)$. Consequently, $\operatorname{dim} \mathcal{N}^{\infty}(S+F) \geq n+1$. But, we have also that $S+F$ is an algebraic operator (see [4, Proposition 3.6]), therefore $S+F \notin \Lambda$. By Lemma 4.1, $\Phi$ is bijective and preserves rank-one operators in both directions. Hence, we obtain that $K=\Phi^{-1}(F)$ is of rank non-greater than $n$ and $I+K \notin \Lambda$. However, $I+K$ is algebraic and $\operatorname{ker}\left((I+K)^{n+1}\right) \subseteq \operatorname{ran}(K)$, and so $I+K \in \Lambda$. This contradiction completes the proof.

Let $\tau$ be a field automorphism of $\mathbb{K}$. An additive map $A: X \rightarrow Y$ between two Banach spaces is called $\tau$-semi linear if $A(\lambda x)=\tau(\lambda) A x$ holds for all $x \in X$ and $\lambda \in \mathbb{K}$. Moreover, we say simply that $A$ is conjugate linear when $\tau$ is the complex conjugation. Notice that if $A$ is non-zero and bounded, then $\tau$ is continuous, and consequently, $\tau$ is either the identity or the complex conjugation (see [9, Theorem 14.4.2 and Lemma 14.5.1]). Moreover, in this case, the adjoint operator $A^{*}: Y^{*} \rightarrow X^{*}$, defined by $A^{*}(g)=\tau^{-1} \circ g \circ A$ for all $g \in Y^{*}$, is again $\tau$-semi linear.

Lemma 4.3. Let $\Phi: \mathcal{B}(X) \rightarrow \mathcal{B}(X)$ be an additive surjective map preserving $\Lambda$ in both directions. Then there exists a non-zero scalar $c$, and either

1. there exists an invertible bounded linear, or conjugate linear, operator $A: X \rightarrow$ $X$ such that $\Phi(F)=c A F A^{-1}$ for all finite-rank operators $F \in \mathcal{B}(X)$, or

2. there exists an invertible bounded linear, or conjugate linear, operator $B: X^{*} \rightarrow$ $X$ such that $\Phi(F)=c B F^{*} B^{-1}$ for all finite-rank operators $F \in \mathcal{B}(X)$. In this case, $X$ is reflexive.

Proof. The existence of a non-zero scalar $c$ such that $\Phi(I)=c I$ is ensured by Lemma 4.2. Clearly, we can suppose without loss of generality that $\Phi(I)=I$. Since $\Phi$ is bijective and preserves the set of rank-one operators in both directions, then by $[16$, Theorems 3.1 and 3.3], there exist a ring automorphism $\tau: \mathbb{K} \rightarrow \mathbb{K}$ and either two bijective $\tau$-semi linear mappings $A: X \rightarrow X$ and $C: X^{*} \rightarrow X^{*}$ such that

$$
\Phi(x \otimes f)=A x \otimes C f \quad \text { for all } x \in X \text { and } f \in X^{*},
$$

or two bijective $\tau$-semi linear mappings $B: X^{*} \rightarrow X$ and $D: X \rightarrow X^{*}$ such that

$$
\Phi(x \otimes f)=B f \otimes D x \quad \text { for all } x \in X \text { and } f \in X^{*} .
$$

Suppose that $\Phi$ satisfies (4.1), and let us show that

$$
C(f)(A x)=\tau(f(x)) \quad \text { for all } x \in X \text { and } f \in X^{*} .
$$

Clearly, it suffices to establish that for all $x \in X$ and $f \in X^{*}, f(x)=-1$ if and only if $C(f)(A x)=-1$. Let $x \in X$ and $f \in X^{*}$. We can choose linearly independent vectors 
$z_{1}, \ldots, z_{n}$ in $\operatorname{ker}(f) \cap \operatorname{ker}(C(f) A)$. Then, it follows from [17, Lemma 3.8] that

$$
\begin{aligned}
f(x)=-1 & \Leftrightarrow \quad \exists\left\{g_{i}\right\}_{i=1}^{n} \subseteq X^{*}: I+x \otimes f+\sum_{i=1}^{n} z_{i} \otimes g_{i} \notin \mathcal{B}_{n}(X) \\
& \Leftrightarrow \quad \exists\left\{g_{i}\right\}_{i=1}^{n} \subseteq X^{*}: I+x \otimes f+\sum_{i=1}^{n} z_{i} \otimes g_{i} \notin \Lambda \\
& \Leftrightarrow \quad \exists\left\{g_{i}\right\}_{i=1}^{n} \subseteq X^{*}: I+A x \otimes C f+\sum_{i=1}^{n} A z_{i} \otimes C g_{i} \notin \Lambda \\
& \Leftrightarrow \exists\left\{g_{i}\right\}_{i=1}^{n} \subseteq X^{*}: I+A x \otimes C f+\sum_{i=1}^{n} A z_{i} \otimes C g_{i} \notin \mathcal{B}_{n}(X) \\
& \Leftrightarrow C(f)(A x)=-1 .
\end{aligned}
$$

Thus, relation (4.3) holds, and arguing as in [16], we get that $\tau, A, C$ are continuous, $\tau$ is the identity or the complex conjugation, and $C=\left(A^{-1}\right)^{*}$. Therefore, $\tau^{-1}=\tau$ and, for every $u \in X$, we have

$$
\Phi(x \otimes f) u=\tau\left(f A^{-1} u\right) A x=A\left(f\left(A^{-1} u\right) x\right)=A(x \otimes f) A^{-1} u .
$$

Thus, $\Phi(x \otimes f)=A(x \otimes f) A^{-1}$ for all $x \in X$ and $f \in X^{*}$; that is, $\Phi(F)=A F A^{-1}$ for all finite-rank operators $F \in \mathcal{B}(X)$.

Now suppose that $\Phi$ satisfies (4.2), and let us show that

$$
D(x)(B f)=\tau(f(x)) \quad \text { for all } x \in X \text { and } f \in X^{*} .
$$

Let $x \in X$ and $f \in X^{*}$. Choose linearly independent linear forms $h_{1}, \ldots, h_{n} \in X^{*}$ such that $h_{i}(x)=0$ and $D(x)\left(B h_{i}\right)=0$ for $1 \leq i \leq n$. Then, it follows from the surjectivity of $D$ and from [17, Lemma 3.8] that

$$
\begin{aligned}
D(x)(B f)=-1 & \Leftrightarrow \exists\left\{u_{i}\right\}_{i=1}^{n} \subseteq X: I+B f \otimes D x+\sum_{i=1}^{n} B h_{i} \otimes D u_{i} \notin \mathcal{B}_{n}(X) \\
& \Leftrightarrow \quad \exists\left\{u_{i}\right\}_{i=1}^{n} \subseteq X: I+B f \otimes D x+\sum_{i=1}^{n} B h_{i} \otimes D u_{i} \notin \Lambda \\
& \Leftrightarrow \quad \exists\left\{u_{i}\right\}_{i=1}^{n} \subseteq X: I+x \otimes f+\sum_{i=1}^{n} u_{i} \otimes h_{i} \notin \Lambda \\
& \Leftrightarrow \exists\left\{u_{i}\right\}_{i=1}^{n} \subseteq X: I+x \otimes f+\sum_{i=1}^{n} u_{i} \otimes h_{i} \notin \mathcal{B}_{n}(X) \\
& \Leftrightarrow \exists\left\{u_{i}\right\}_{i=1}^{n} \subseteq X: I+f \otimes \mathrm{J} x+\sum_{i=1}^{n} h_{i} \otimes \mathrm{J} u_{i} \notin \mathcal{B}_{n}\left(X^{*}\right) \\
& \Leftrightarrow f(x)=-1,
\end{aligned}
$$

where $\mathrm{J}: X \rightarrow X^{* *}$ is the natural embedding. Thus, relation (4.4) holds, and arguing as in [16], we get that $\tau, B, D$ are continuous, $\tau$ is the identity or the complex conjugation, and $D=\left(B^{-1}\right)^{*} \mathrm{~J}$. But, the operators $D$ and $\left(B^{-1}\right)^{*}$, and therefore also 
$\mathrm{J}$ are bijections, which implies the reflexivity of $X$. Furthermore, $\tau^{-1}=\tau$ and, for every $u \in X$, we have

$$
\begin{aligned}
\Phi(x \otimes f) u & =\left(B f \otimes\left(B^{-1}\right)^{*} \mathrm{~J}(x)\right) u=\left(B^{-1}\right)^{*} \mathrm{~J}(x)(u) \cdot B f \\
& =\tau\left(\mathrm{J}(x)\left(B^{-1} u\right)\right) \cdot B f=B\left(\mathrm{~J}(x)\left(B^{-1} u\right) f\right) \\
& =B(f \otimes \mathrm{J}(x)) B^{-1} u=B(x \otimes f)^{*} B^{-1} u .
\end{aligned}
$$

Thus, $\Phi(x \otimes f)=B(x \otimes f)^{*} B^{-1}$ for all $x \in X$ and $f \in X^{*}$. Hence, $\Phi(F)=B F^{*} B^{-1}$ for all finite-rank operator $F \in \mathcal{B}(X)$. This completes the proof.

Theorem 4.4. Let $\Phi: \mathcal{B}(X) \rightarrow \mathcal{B}(X)$ be an additive surjective map preserving $\Lambda$ in both directions. Then there exists a non-zero scalar $c$, and either

1. there exists an invertible bounded linear, or conjugate linear, operator $A: X \rightarrow$ $X$ such that $\Phi(T)=c A T A^{-1}$ for all $T \in \mathcal{B}(X)$, or

2. there exists an invertible bounded linear, or conjugate linear, operator $B: X^{*} \rightarrow$ $X$ such that $\Phi(T)=c B T^{*} B^{-1}$ for all $T \in \mathcal{B}(X)$.

Proof. Since $\Phi$ preserves $\Lambda$ in both directions, it follows that $\Phi$ takes one of the two forms in Lemma 4.3.

Suppose that $\Phi(F)=c A F A^{-1}$ for all finite-rank operators $F \in \mathcal{B}(X)$. Let

$$
\Psi(T)=c^{-1} A^{-1} \Phi(T) A \quad \text { for all } T \in \mathcal{B}(X) .
$$

Clearly, $\Psi$ satisfies the same properties as $\Phi$. Furthermore, $\Psi(I)=I$ and $\Psi(F)=F$ for all finite-rank operators $F \in \mathcal{B}(X)$. Let $T \in \mathcal{B}(X)$ and choose an arbitrary rational number $\lambda$ such that $T-\lambda$ and $\Psi(T)-\lambda$ are invertible. Let $F \in \mathcal{B}(X)$ be a finite-rank operator. Since $T-\lambda+F$ and $\Psi(T)-\lambda+F$ are Fredholm of index zero, then

$$
\begin{aligned}
T-\lambda+F \in \mathcal{B}_{n}(X) & \Leftrightarrow T-\lambda+F \in \Lambda \Leftrightarrow \Psi(T)-\lambda+F \in \Lambda \\
& \Leftrightarrow \Psi(T)-\lambda+F \in \mathcal{B}_{n}(X) .
\end{aligned}
$$

Hence, we get by $[17$, Proposition 2.17] that $\Psi(T)=T$.

This shows that $\Phi(T)=c A T A^{-1}$ for all $T \in \mathcal{B}(X)$.

Now suppose that $\Phi(F)=c B F^{*} B^{-1}$ for all finite-rank operators $F \in \mathcal{B}(X)$. Then Lemma 4.3 ensures that $X$ is reflexive. By considering

$$
\Gamma(T)=c^{-1} \mathrm{~J}^{-1}\left(B^{-1} \Phi(T) B\right)^{*} \mathrm{~J} \quad \text { for all } T \in \mathcal{B}(X),
$$

we get in a similar way that $\Gamma(T)=T$ for all $T \in \mathcal{B}(X)$. Thus, $\Phi(T)=c B T^{*} B^{-1}$ for all $T \in \mathcal{B}(X)$, as desired. This finishes the proof.

With these results at hand, we are ready to prove our main results.

Proof of Theorem 1.1. (1) $\Rightarrow$ (4). Suppose that $\Phi$ preserves $\mathcal{A}_{n}(H)$ in both directions. Using the fact that $\Phi$ is surjective, it follows by Theorem 2.7 that, for every $T \in \mathcal{B}(H)$,

$$
\begin{aligned}
T & \in \mathcal{B}_{n}^{+}(H) \Leftrightarrow \forall S \in \mathcal{B}(H), \exists \varepsilon_{0}>0:\left\{T+\varepsilon S: \varepsilon \in \mathbb{Q} \text { and }|\varepsilon|<\varepsilon_{0}\right\} \subseteq \mathcal{A}_{n}(H) \\
& \Leftrightarrow \quad \forall S \in \mathcal{B}(H), \exists \varepsilon_{0}>0:\left\{\Phi(T)+\varepsilon \Phi(S): \varepsilon \in \mathbb{Q} \text { and }|\varepsilon|<\varepsilon_{0}\right\} \subseteq \mathcal{A}_{n}(H) \\
& \Leftrightarrow \quad \forall R \in \mathcal{B}(H), \exists \varepsilon_{0}>0:\left\{\Phi(T)+\varepsilon R: \varepsilon \in \mathbb{Q} \text { and }|\varepsilon|<\varepsilon_{0}\right\} \subseteq \mathcal{A}_{n}(H) \\
& \Leftrightarrow \Phi(T) \in \mathcal{B}_{n}^{+}(H) .
\end{aligned}
$$


Thus $\Phi$ preserves $\mathcal{B}_{n}^{+}(H)$ in both directions. It follows that $\Phi$ takes one of the two forms in Theorem 4.4. Let us show that $\Phi$ cannot take the form

$$
\Phi(T)=c B T^{*} B^{-1} \quad \text { for all } T \in \mathcal{B}(H) .
$$

Suppose on the contrary that $\Phi$ takes the form (4.5). Let $\left\{e_{n}: n \geq 0\right\}$ be an arbitrary orthonormal basis of $H$. Consider the weighted unilateral shift operator $U \in \mathcal{B}(H)$ given by

$$
U e_{n}=(n+1)^{-1} e_{n+1} \text { for every } n \geq 0 .
$$

Clearly, $U$ is an injective quasi-nilpotent operator.

Thus, $\mathrm{a}\left(U^{*}\right)=\mathrm{d}\left(U^{*}\right)=\infty, U \in \mathcal{B}_{n}^{+}(H)$ and $U^{*} \notin \mathcal{B}_{n}^{ \pm}(H)$.

So that $\Phi(U)=c B U^{*} B^{-1} \notin \mathcal{B}_{n}^{ \pm}(H)$, a contradiction.

$(2) \Rightarrow(4)$. Now, suppose that $\Phi$ preserves $\mathcal{D}_{n}(H)$ in both directions. As above, using Theorem 2.7 we infer that $\Phi$ preserves $\mathcal{B}_{n}^{-}(H)$ in both directions, and so $\Phi$ takes one of the two forms in Theorem 4.4. Consider the unilateral shift operator $S \in \mathcal{B}(H)$ given by

$$
S e_{0}=0 \quad \text { and } \quad S e_{n}=e_{n-1} \text { for } n \geq 1 .
$$

Clearly, $S$ is surjective and a $(S)=\infty$.

Thus, $\mathrm{d}\left(S^{*}\right)=\infty, S \in \mathcal{B}_{n}^{-}(H)$ and $S^{*} \notin \mathcal{B}_{n}^{-}(H)$. This contradiction shows that $\Phi$ cannot take the form (4.5).

$(3) \Rightarrow(4)$ is similar to the first implication with the same example (4.6).

$(4) \Rightarrow(1),(2)$ and $(3)$ are obvious.

Proof of Theorem 1.2. Follows from Theorems 2.5 and 4.4.

Proof of Corollary 1.3. The proof is similar to the proof of Theorem 1.1.

\section{References}

[1] Aupetit, B., Spectrum-preserving linear mappings between Banach algebras or JordanBanach algebras, J. London Math. Soc., 62(2000), 917-924.

[2] Aupetit, B., A Primer on Spectral Theory, Springer-Verlag, New York, 1991.

[3] Bel Hadj Fredj, O., Burgos, M., Oudghiri, M., Ascent and essential ascent spectrum, Studia Math., 187(2008), 59-73.

[4] Burgos, M., Kaidi, A., Mbekhta, M., Oudghiri, M., The descent spectrum and perturbations, J. Operator Theory, 56(2006), 259-271.

[5] Grabiner, S., Zemánek, J., Ascent, descent, and ergodic properties of linear operators, J. Operator Theory, 48(2002), 69-81.

[6] Hou, J., Cui, J., Additive maps on standard operator algebras preserving invertibilities or zero divisors, Linear Algebra Appl., 359(2003), 219-233.

[7] Jafarian, A.A., Sourour, A.R., Spectrum-preserving linear maps, J. Funct. Anal., 66(1986), 255-261.

[8] Kaplansky, I., Algebraic and Analytic Aspects of Operator Algebras, Amer. Math. Soc. Providence, RI, 1970.

[9] Kuczma, M., An Introduction to the Theory of Functional Equations and Inequalities, Państwowe Wydawnictwo Naukowe, Warszawa, 1985. 
[10] Marcus, M., Purves, R., Linear transformations on algebras of matrices: The invariance of the elementary symmetric functions, Canad. J. Math., 11(1959), 383-396.

[11] Mbekhta, M., Müller, V., Oudghiri, M., Additive preservers of the ascent, descent and related subsets, J. Operator Theory, 71(2014), 63-83.

[12] Mbekhta, M., Müller, V., Oudghiri, M., On additive preservers of semi-Browder operators, Rev. Roumaine Math. Pures Appl., 59(2014), 237-244.

[13] Mbekhta, M., Oudghiri, M., On additive maps preserving certain semi-Fredholm subsets, Linear Multilinear Algebra, 61(2012), 1010-1016.

[14] Mbekhta, M., Šemrl, P., Linear maps preserving semi-Fredholm operators and generalized invertibility, Linear Multilinear Algebra, 57(2009), 55-64.

[15] Müller, V., Spectral Theory of Linear Operators and Spectral Systems in Banach Algebras, Second edition, Operator Theory: Advances and Applications, 139, Birkhäuser Verlag, Basel, 2007.

[16] Omladič, M., Šemrl, P., Additive mappings preserving operators of rank one, Linear Algebra Appl., 182(1993), 239-256.

[17] Oudghiri, M., Souilah, K., Additive preservers of Drazin invertible operators with bounded index, Acta Math. Sin. (Engl. Ser.), 33(2017), 1225-1241.

[18] Taylor, A.E., Theorems on Ascent, Descent, Nullity and Defect of Linear Operators, Math. Ann., 163(1966), 18-49.

[19] Taylor, A.E., Lay, D.C., Introduction to Functional Analysis, Wiley, New YorkChichester-Brisbane, 1980.

Mourad Oudghiri

Université Mohammed Premier

Département Math, Labo LAGA, Faculté des Sciences d'Oujda

60000 Oujda, Maroc

e-mail: morad.oudghiri@gmail.com

Khalid Souilah

Université Mohammed Premier

Département Math, Labo LAGA, Faculté des Sciences d'Oujda

60000 Oujda, Maroc

e-mail: s.khalide@gmail.com 